\title{
Fluoropolymer/GAP Block Copolyurethane Binders: Sensitivity, Mechanical Properties and Reactive Properties with Aluminum
}

\section{Minghui Xu ( $\sim$ mhuixu@163.com)}

Xi'an Modern Chemistry Research Institute

Xianming Lu

Xi'an Modern Chemistry Research Institute

Ning Liu

Xi'an Modern Chemistry Research Institute

Qian Zhang

Xi'an Modern Chemistry Research Institute

Hongchang Mo

Xi'an Modern Chemistry Research Institute

Zhongxue Ge

Xi'an Modern Chemistry Research Institute

\section{Research Article}

Keywords: olid propellant, energetic copolyurethane binder, PBFMO, drop weight test, XPS, tensile test, SEM, DSC

Posted Date: February 16th, 2021

DOI: https://doi.org/10.21203/rs.3.rs-199382/v1

License: (c) (1) This work is licensed under a Creative Commons Attribution 4.0 International License. Read Full License 


\section{Fluoropolymer/GAP block copolyurethane binders: Sensitivity,}

\section{2 mechanical properties and reactive properties with aluminum}

3 MinghuiXu, ${ }^{\mathrm{a}, \mathrm{b},{ }^{*}}$ Xianming Lu, ${ }^{\mathrm{b}}$ Ning Liu, ${ }^{\mathrm{b}}$ Qian Zhang, ${ }^{\mathrm{b}}$ Hongchang Mo, ${ }^{\mathrm{b}}$ and Zhongxue $\mathrm{Ge}^{\mathrm{b}}$

${ }^{a}$ State Key Laboratory of Fluorine \& Nitrogen Chemicals, Xi'an 710065 (P. R. China)

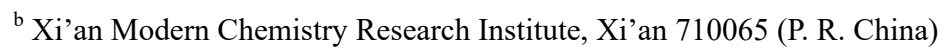

Email: mhuixu@163.com

Abstract: In order to enhance the application properties of GAP in solid propellant, an energetic copolyurethane binder, (poly[3,3-bis(2,2,2-trifluoro-ethoxymethyl)oxetane] glycol-block-glycidylazide polymer (PBFMO-b-GAP) was developed. The PBFMO- $b$-GAP was prepared using poly[3,3-bis(2,2,2-trifluoro-ethoxymethyl)oxetane] glycol (PBFMO) which preparing from cationic polymerization and GAP as the raw materials, TDI as the coupling agent via a prepolymer process. The molecular structure of copolyurethane was confirmed by FT-IR, NMR, GPC. The impact sensitivity, mechanical properties and thermal behavior of PBFMO- $b$-GAP were studied by drop weight test, XPS, tensile test, SEM, DSC and TG/DTG respectively. The results proved that the introduction of fluoropolymer can evidently reduce the sensitivity of GAP based polyurethanes and enhance their mechanical behavior (the tensile strength up to $5.75 \mathrm{MPa}$ with a breaking elongation of $1660 \%$ ). Also, PBFMO-b-GAP exhibited an excellent resistance to thermal decomposition up to $200^{\circ} \mathrm{C}$ and good compatibility with $\mathrm{Al}$ and $\mathrm{HMX}$. Cook-off test was used to investigate the reactive of copolyurethanes and $\mathrm{Al}$, the results indicated that the copolyurethanes could react with $\mathrm{Al}$ efficiently and release significantly more heat. Therefore, the energetic copolyurethanes may serve as promising energetic binders for future propellant formulations.

\section{1.Introduction}

21 A recent trend in the field of energetic material formulations (explosives/propellants) is to replace inert binders (viz., HTPB, CTPB, HTPE, etc.) by energetic binders, which contain energetic groups 
such as $-\mathrm{N}_{3}$ (azide), nitro (C-nitro, $\mathrm{O}$-nitro (nitrate ester), $\mathrm{N}$-nitro (nitramine) and difluroamine

24 groups, to impart additional energy to the system $^{1-3}$. Among energetic polymers, glycidyl azide polymer (GAP) has been extensively studied as polymeric binders since it was first reported in a patent in 1972 by Vandenburg ${ }^{4-6}$. Due to its high density with positive heat of formation of +117.2 kcal mol ${ }^{-1}$, high density $\left(1.3 \mathrm{~g} \mathrm{~cm}^{-3}\right)$, low glass-transition temperature $\left(\mathrm{Tg}=-45^{\circ} \mathrm{C}\right)$, good thermally stability, low detonation tendency, and also high burning rate $\left(\left(1 \mathrm{~cm} \mathrm{~s}^{-1} \text { at } 40 \text { atmospheres }\right)^{7,8}\right.$. GAP has become the hotspot in the field of energy materials by offering a unique energetic binder and plasticizer system for advanced propellants and plastic bonded explosives (PBX) for achieving higher performance ${ }^{9}$. However, traditional GAP-based binders are thermosetpolymer and usually difficult to recycled. Moreover, it also suffers from high sensitive and inferior mechanical behavior which due to their highly polarity of azide groups and poor flexibility of polymer backbone ${ }^{10}$.

In order to overcome these difficulties and also to obtain a better performance, various energetic polymeric binders have been developed in the last two decades. Energetic thermoplastic elastomers (ETPE) as high performance recyclable polymeric binders have received widespread attention in the past decades ${ }^{11}$. Generally, ETPE as a multiblock copolymer consists of hard segments and soft segments. Under room temperature, the hard segments, which act as fillers and physical crosslinks, are in a crystalline state or amorphous glassy state while the soft segments are in a rubbery state that leads to the flexibility. These polymers exhibit excellent mechanical and recyclable properties owing to the phase separation between hard and soft blocks and reversibly cross-linking points ${ }^{12,13}$. Thus, development of novel ETPE has attracted extensive attentions of many researchers in recent years $^{14,15}$

In the past three decades, fluoropolymers have gained considerable attention in the energetic 
material community (such as aerial infrared decoys, igniters, tracking flares, reactive binder systems, and solid fuel rocket propellants) as high explosive binders, owing to their high densities, long-term chemical stabilities, low coefficients of friction, and good compatibility with the main ingredients (oxidizers, metal fuels and plasticizer) ${ }^{16-18}$. Particularly, fluoropolymers can release high reaction energy due to their strong oxidation ${ }^{19-21}$, for instance, the magnesium, Teflon, and Viton system (MTV) as one of the well-known compositions used in decoys and flares, can release especially large specific reaction energy of $9.4 \mathrm{~kJ} \mathrm{~g}^{-1}$, in comparison with TNT and RDX yield just $3.72 \mathrm{~kJ} \mathrm{~g}^{-1}$ and $6.569 \mathrm{~kJ} \mathrm{~g}^{-1}$, respectively ${ }^{22}$. Therefore, synthesis of fluorine-containing GAP based ETPE may be a promising method to improve its performance of application. prepolymer process by coupling together poly[3,3-bis(2,2,2-trifluoroethoxymethyl) oxetane] glycol (PBFMO) and GAP to decrease the sensitivity, enhance the mechanical properties and also promote the reactive efficiency with Al. The chemical structure and molecular weight of copolyurethanes were characterized by FTIR, NMR, GPC. The impact sensitivity and mechanical properties of the copolyurethanes were tested by drop weight test, XPS, tensile test and SEM respectively. The thermal properties of copolyurethanes and their compatibility with Al, HMX were also described by differential scanning calorimetry (DSC), thermal gravimetric analysis (TGA). The reactivity of PBFMO- $b$-GAP/Al complex was investigated by cook-off test.

\section{Results and discussion}



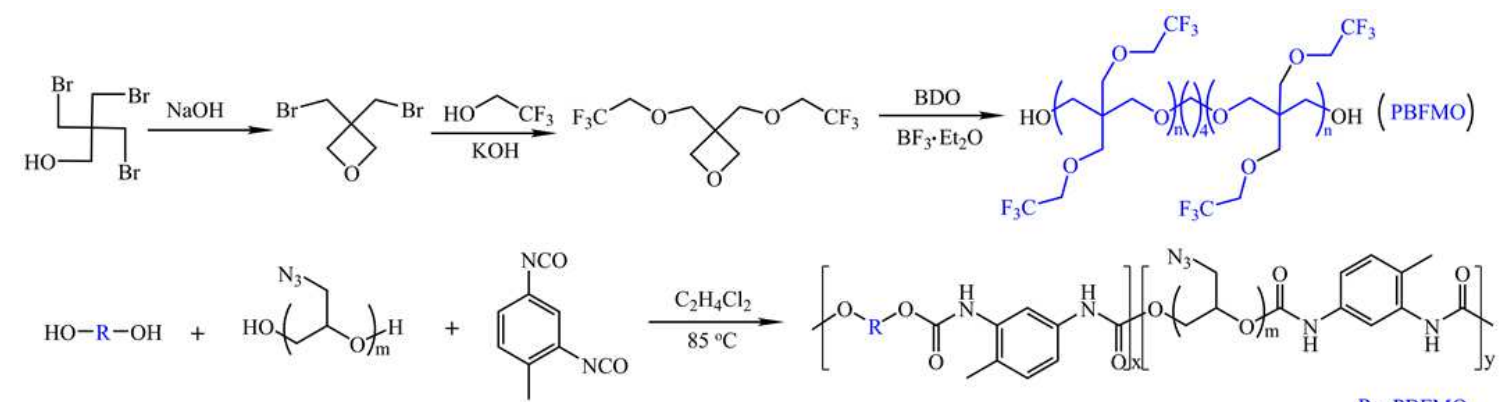

Scheme 1. The synthesis route of PBFMO-b-GAP copolyurethane

2.1 Preparation of PBFMO-b-GAP copolyurethane

67 The synthesis of PBFMO- $b$-GAP copolyurethane was done via a prepolymer process using PBFMO and GAP as the raw materials, TDI as the coupling agent, which was described in Scheme 1. The structure of the as-synthesized PBFMO- $b$-GAP was confirmed by FTIR and NMR. The IR spectra gave a first confirmation of the copolymer structure. As shown in Figure 1, the characteristic adsorption peak at $1276 \mathrm{~cm}^{-1}$ explained the $-\mathrm{CF}_{3}$ stretching vibration of PBFMO, and the bands around $1135 \mathrm{~cm}^{-1}$ accounted for the $\mathrm{C}-\mathrm{O}$ group stretching vibration of PBFMO ${ }^{23,24}$. The appearance

73 of strong peak at $2093 \mathrm{~cm}^{-1}$ were attributed to $-\mathrm{N}_{3}$ from GAP and the appearance of $3320 \mathrm{~cm}^{-1}$ was

74 due to the $-\mathrm{NH}$ stretching vibration, the appearance of $1726 \mathrm{~cm}^{-1}, 1531$ and $1376 \mathrm{~cm}^{-1}$ were assigned 75 to $\mathrm{C}=\mathrm{O}$ and $-\mathrm{NH}$ stretching bands of urethane group ${ }^{25,26}$. Therefore, such results gave strong evidence that the reaction of PBFMO with GAP indeed by the formation of PBFMO- $b$-GAP

77 polyurethane. 


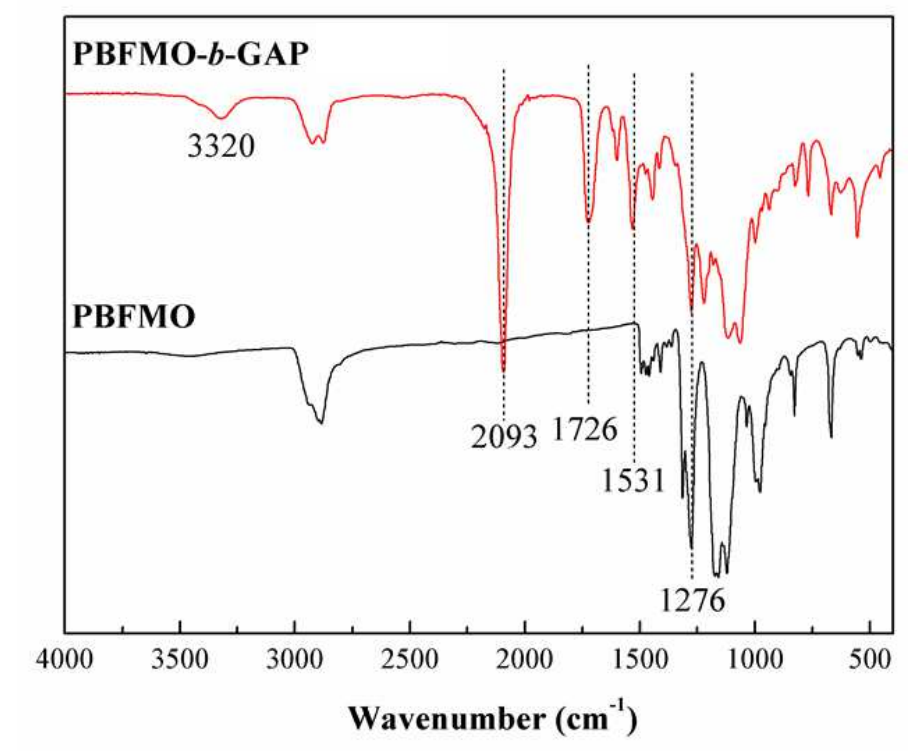

Figure 1. FTIR spectra of (A) PBFMO and (B) PBFMO- $b$-GAP copolyurethane.

As shown in Figure 2A, the signals of a broad band at $3.62 \mathrm{ppm}$ attributed to methylene protons of GAP and PBFMO, and the peak appeared at $3.33 \mathrm{ppm}$ was correspond to methylene protons of PBFMO side chain. The corresponding ${ }^{13} \mathrm{C}-\mathrm{NMR}$ (Figure 2B) also indicated the presence of all the carbons in the GAP and PBFMO. The correspond carbons signals of methylene carbons from GAP and PBFMO appeared at 69.5 and $77.8 \mathrm{ppm}$. The signal for $51.6 \mathrm{ppm}$ is attributed to the quaternary carbon atom of $\mathrm{C}-\mathrm{N}_{3}{ }^{27}$. The signal at $2.17 \mathrm{ppm}$ belongs to methylene protons of the BDO which was the initiator of PBFMO and GAP, the corresponding carbon signals were appeared at $25.7 \mathrm{ppm}$. The signal at $1.77 \mathrm{ppm}$ and $6.5-8 \mathrm{ppm}$ were attributed to the methyl protons and methine protons on benzene rings of the TDI, respectively, and the corresponding carbons signals were appeared at 17.1 ppm and 120-135 ppm. Moreover, as shown in Figure 2C of ${ }^{19} \mathrm{~F}$ NMR, the peak at -74.4 ppm was 90 attributed to the $-\mathrm{CF}_{3}$ of the side chain ${ }^{28}$. These signal positions observed in the NMR spectra of PBFMO- $b$-GAP strictly corroborated our FTIR analysis results. 

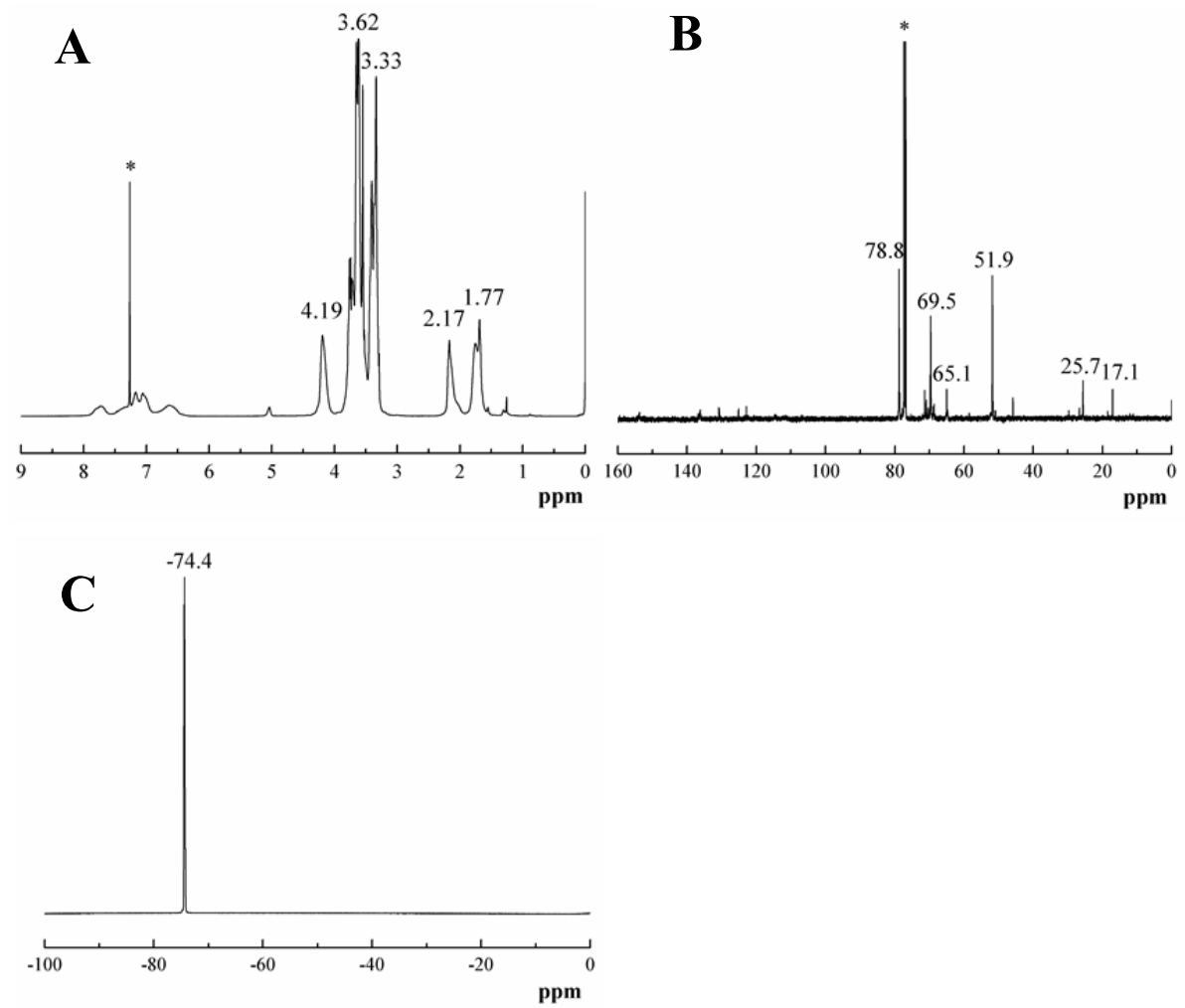

Figure 2. ${ }^{1} \mathrm{H}-\mathrm{NMR}$ spectra (A), ${ }^{13} \mathrm{C}-\mathrm{NMR}$ spectra (B) and ${ }^{19} \mathrm{~F}-\mathrm{NMR}$ spectra $(\mathrm{C})$ of PBFMO- $b$-GAP in $\mathrm{CDCl}_{3}$.

To investigate the different PBFMO- $b$-GAP copolyurethanes, the molar ratio of PBFMO/GAP was set at $1 / 3,1 / 9$ and $1 / 19$, during chain coupling by TDI to obtain PBFMO- $b$-GAP- $1^{\#}, 2^{\#}$ and $3^{\#}$ respectively. As showed in Table 1, the density of PBFMO- $b$-GAP was around 1.273 1.308 $\mathrm{g} \mathrm{cm}^{-3}$, which is higher than that of the control group (GAP based polyurethane, GAP-ETPE $1.263 \mathrm{~g} \mathrm{~cm}^{-3}$ ). It is well known that polymers containing $-\mathrm{CF}_{3}$ group increase its density, which is seen in the present study also ${ }^{29}$. The impact sensitivity of energetic polymer gels were characterized by the drop weight test, and the results are also showed in Table 1. It can be seen that the sensitivity of energetic materials is reduced with the mass ratio of polymer in the gel increases. Particularly noteworthy is the fact that the PBFMO/GAP molar ratio at 1/19 is markedly less sensitive than the pure GAP based polyurethane. Therefore, it appeared that the introduction of fluoropolymer may be manipulated to 
reduce the sensitivity of very high energy composite energetic materials made in this fashion.

Table 1. Relative molecular mass, density and $H_{50}$ of GAP based polyurethanes in different molar ratios

\begin{tabular}{ccccc}
\hline Sample & PBFMO/GAP molar ratio & $\mathrm{M}_{\mathrm{n}}\left(10^{3} \mathrm{gmol}^{-1}\right)$ & Density $\left(\mathrm{g} \mathrm{cm}^{-3}\right)$ & $H_{50}(\mathrm{~cm})$ \\
\hline PBFMO- $b$-GAP-1 $^{\#}$ & $1 / 3$ & 33 & 1.308 & $>129$ \\
PBFMO- $b$-GAP-2 $2^{\#}$ & $1 / 9$ & 31 & 1.290 & $>129$ \\
PBFMO- $b$-GAP-3 & $1 / 19$ & 30 & 1.273 & 56.2 \\
GAP-ETPE & 0 & 32 & 1.263 & 9.55 \\
\hline
\end{tabular}

XPS was employed to detect the change of elementary composition on the elastomers surface, and provide valuable insight into the influence between sensitivity and fluorine content ${ }^{30}$. The N1s, C1s, O1s and F1s elements of XPS spectra of elastomers surface and its surface compositions expressed quantitatively as atomic weight percentages are summarized in Figure 3and Table 2, respectively. Due to the introduction of different content of PBFMO, the concentrations of $F$ from elastomers surface increase from 0 to $13.05 \%$, meanwhile the atomic weight percentage of $\mathrm{N}$

114 decrease from $12.16 \%$ to $1.54 \%$. The results indicated that the increase of the F elements of the surface may decrease of the coefficients of friction of the atomic weight percentage and decrease its sensitivity.
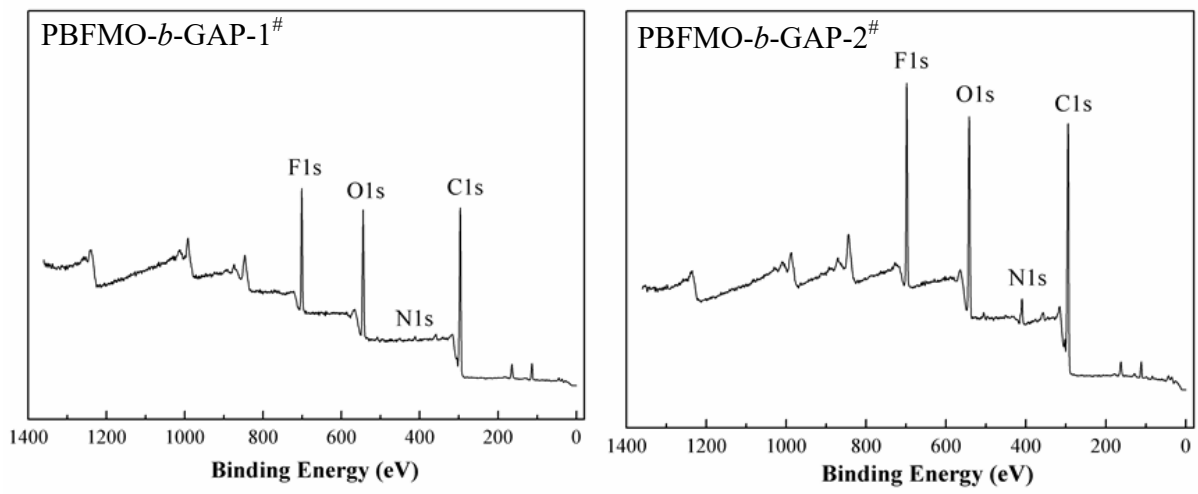

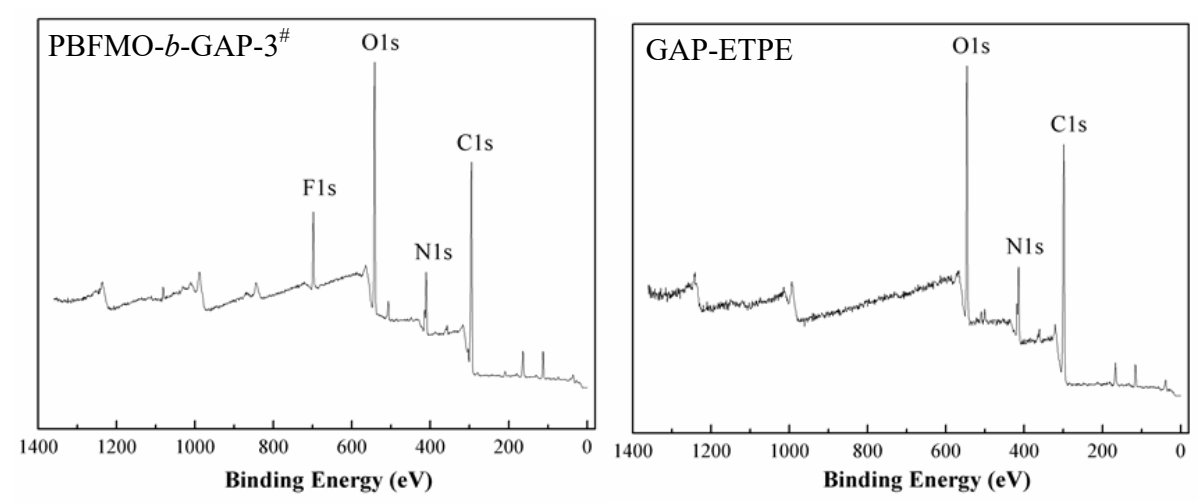

Figure 3. XPS curves of the gels prepared from GAP based polyurethanes

Table 2. Atomic weight percentages of XPS cruves

\begin{tabular}{|c|c|c|c|c|}
\hline Sample & $\mathrm{C}(\%)$ & $\mathrm{O}(\%)$ & $\mathrm{N}(\%)$ & $\mathrm{F}(\%)$ \\
\hline PBFMO- $b$-GAP-1 ${ }^{\#}$ & 65.22 & 20.18 & 1.54 & 13.05 \\
\hline PBFMO- $b$-GAP-2 ${ }^{\#}$ & 63.00 & 19.76 & 4.33 & 12.91 \\
\hline PBFMO- $b$-GAP- $3^{\#}$ & 60.88 & 23.42 & 10.36 & 5.34 \\
\hline GAP-ETPE & 63.8 & 24.04 & 12.16 & 0 \\
\hline
\end{tabular}

1212.3 Mechanical Properties of PBFMO-b-GAP copolyurethane

122 Mechanical properties of the PBFMO- $b$-GAP copolyurethanes prepared from various ratio of

123 PBFMO/GAP prepolymers were evaluated with the universal testing machine as shown in Figure 4.

124 An overlay of stress-strain curves of PBFMO-b-GAP copolyurethanes shown in Figure 4, showed an 125 initial linear deformation, subsequent extension and ultimately lead to the failure. It is clearly that the 126 tensile strength of PBFMO- $b$-GAP increase from 2.9 to 5.75 MPa along with the increase of PBFMO 127 content, meanwhile the elongation at break decrease from $2056 \%$ to $1660 \%$. In general, the 128 crosslinking density is a dominant factor to determining mechanical performances for 129 network-structured materials, higher crosslinking density results in higher tensile strength and 130 Young's modulus, and lower elongation at break. In this work, PBFMO works as a hard segment in 

segment in GAP based polyurethanes to increase their mechanical behavior.

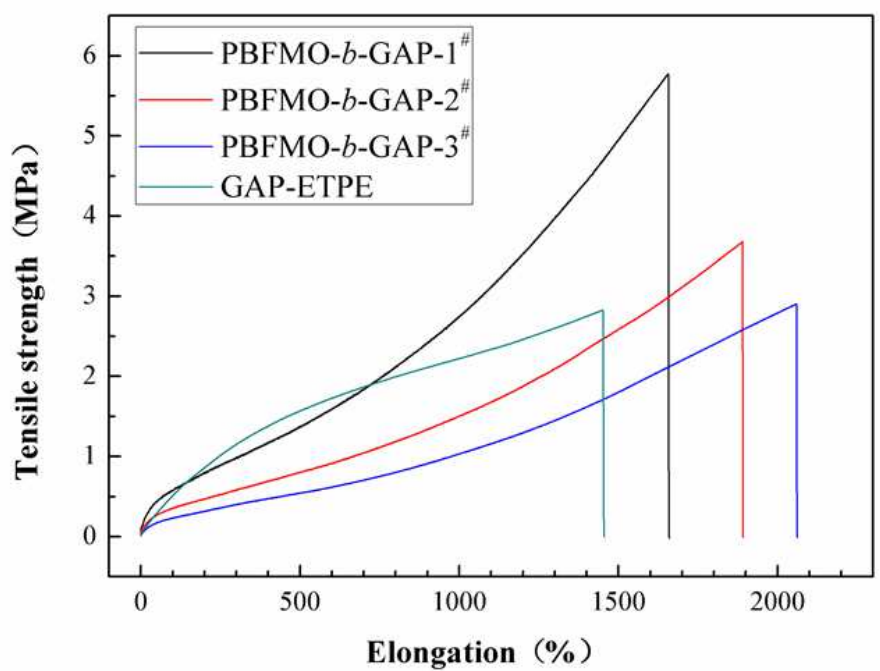

Figure 4.Tensile testing of polymer samples prepared from PBFMO- $b$-GAP copolyurethane.

To get some insight of the observed enhancement of the mechanical properties of the

PBFMO- $b$-GAP copolyurethane, the fractured surfaces of the elastomers films with various molar ratio of GAP/PBFMO were studied by SEM. As shown in Figure 5, with an increase in the molar ratio of GAP/PBFMO, the wrinkle and ravines of the fractured surfaces were more and more obvious while the fractured stripes became deeper. It is well known that phase separation exists between the soft and hard segments, and the hard segments aggregate with each other to form physical cross-linking points throughout the soft segments, resulting in good mechanical properties. The phenomenon might be ascribed to the fact that structural heterogeneity in the films gradually aggravated with an increase of crosslinking densities. It may reveal that the introduction of PBFMO uniformly improved the phase separation of gel prepared from PBFMO- $b$-GAP, and resulted in good mechanical properties ${ }^{31}$. Consideration of PBFMO- $b-$ GAP- $1^{\#}$ which possessing the best tensile strength and sufficient elongation at break, was selected in the next experiment. 

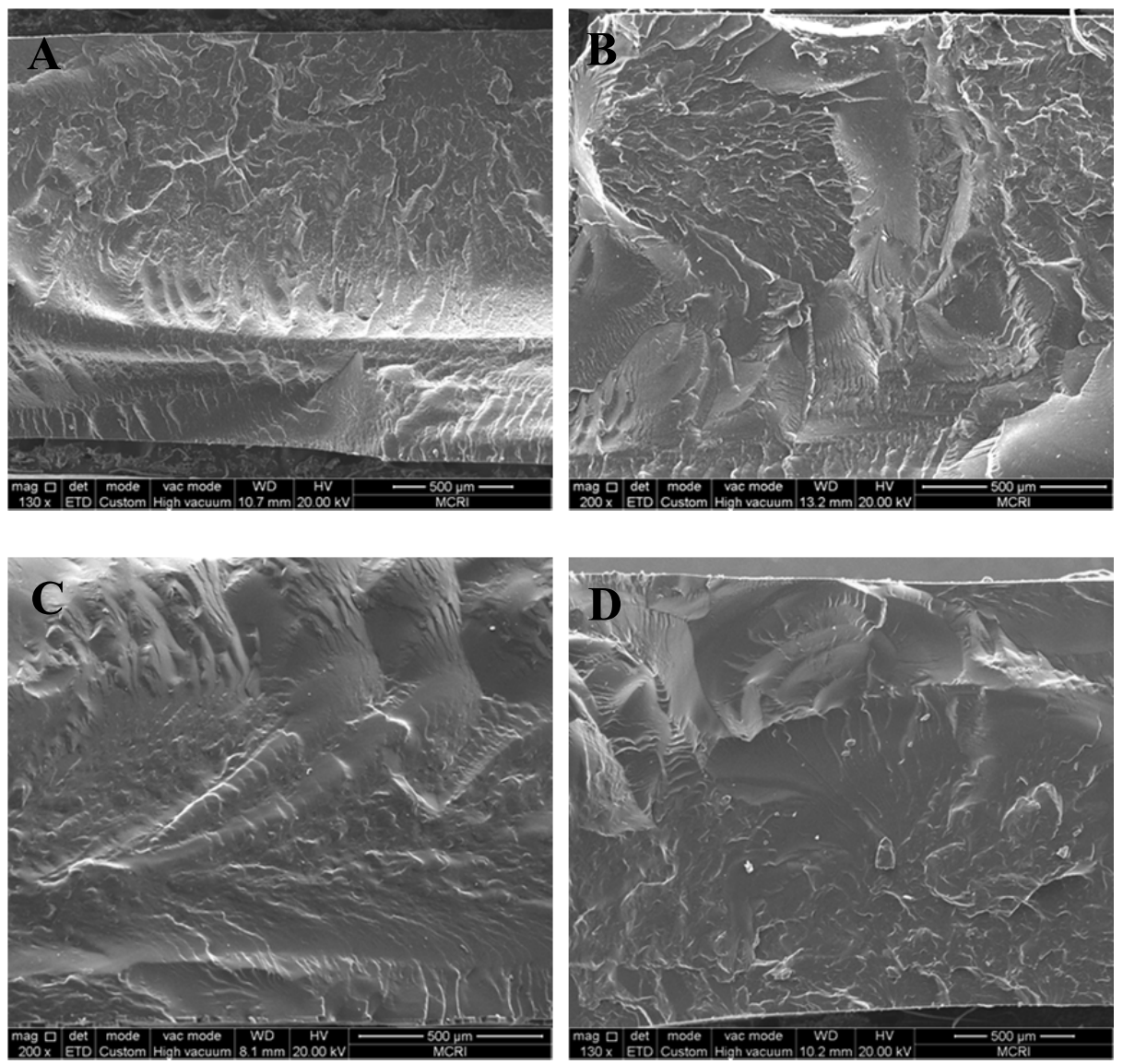

Figure 5. SEM images for the fracture surface of the gels prepared from (A) PBFMO- $b$-GAP-1 $1^{\#}$, (B)

PBFMO- $b-G A P-2^{\#}$, (C) PBFMO- $b-G A P-3^{\#}$ and (D) GAP-ETPE.

\subsection{Thermal decomposition}

153 It is well known that the thermal stability of energetic binders plays an important role in the

154 preparation, processing, storage, and application of energetic material ${ }^{32,33}$. Thus, DSC and TGA were applied to study the thermal decomposition behavior of PBFMO- $b$-GAP copolyurethane. The DSC

showed four exothermic peaks. The first exothermic peak at $40^{\circ} \mathrm{C}$ was the melting point of PBFMO, the second exothermic peak at $247^{\circ} \mathrm{C}$ was caused by the decomposition of side chain azide groups on PBFMO- $b$-GAP to give nitrogen molecules, and the other two peaks at 453 and $504{ }^{\circ} \mathrm{C}$ were due 

around $25 \%$ with respect to the total was at $246{ }^{\circ} \mathrm{C}$, which was corresponding to the stripping of the azide groups of the side chain which in correspondence of the same phenomena of DSC. It is seen

164 from the TGA curve that after the sharp step does not level and shows a gradual weight loss. The 165 phenomenon is superposed to an incipient degradation of the polymer chains. In any case, both DSC and TGA confirm that the PBFMO- $b$-GAP start to decompose/degrade at high temperature, thus showing a satisfactory thermal stability.

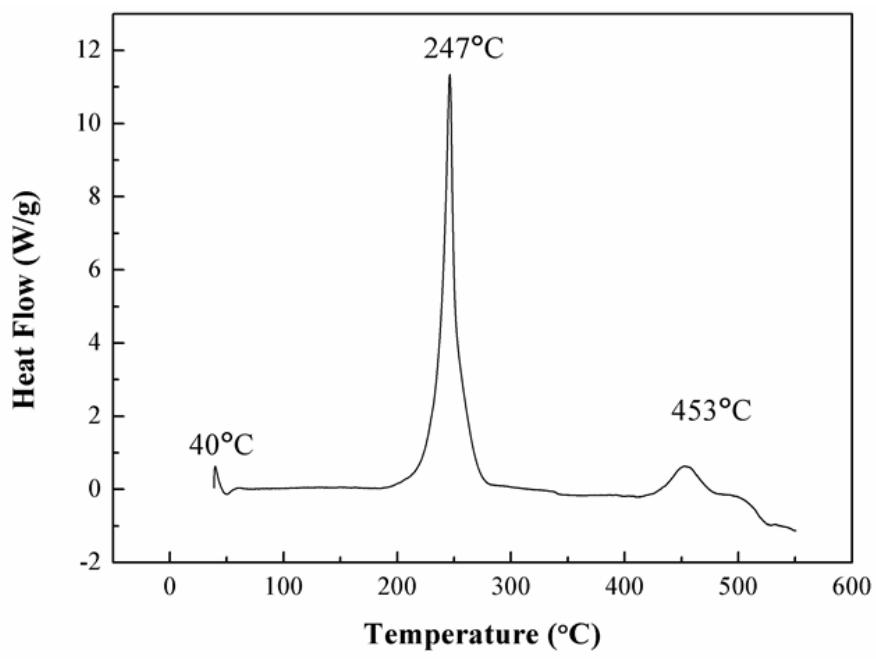

Figure 6. DSC curve of PBFMO- $b$-GAP copolyurethane.

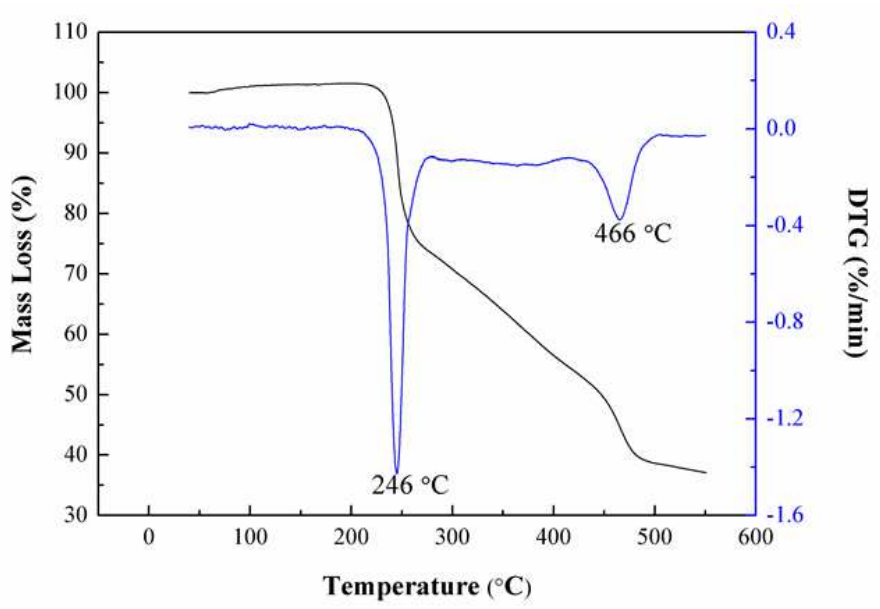




\subsection{Compatibility testing}

Compatibility is an important safety and reliability index used to evaluate the production, application and storage of energetic materials ${ }^{34,35}$. Usually, compatibility can be evaluated from DSC curves by studying the effect of the contact material on the exothermic decomposition temperature of the explosives. In this study, DSC curves were used to determine the compatibility of PBFMO- $b$-GAP with the main energetic components, such as HMX and Al. Typical DSC curves of binary systems PBFMO- $b$-GAP/HMX, PBFMO- $b$-GAP/Al were shown in Figure 8. According to the standards of compatibility, the binary systems PBFMO- $b$-GAP/HMX and PBFMO- $b$-GAP /A1 had good compatibilities because their $\Delta T \mathrm{p}$ values were all less than $2{ }^{\circ} \mathrm{C}$. It indicated that PBFMO- $b$-GAP could be safely used in HMX based propellant.

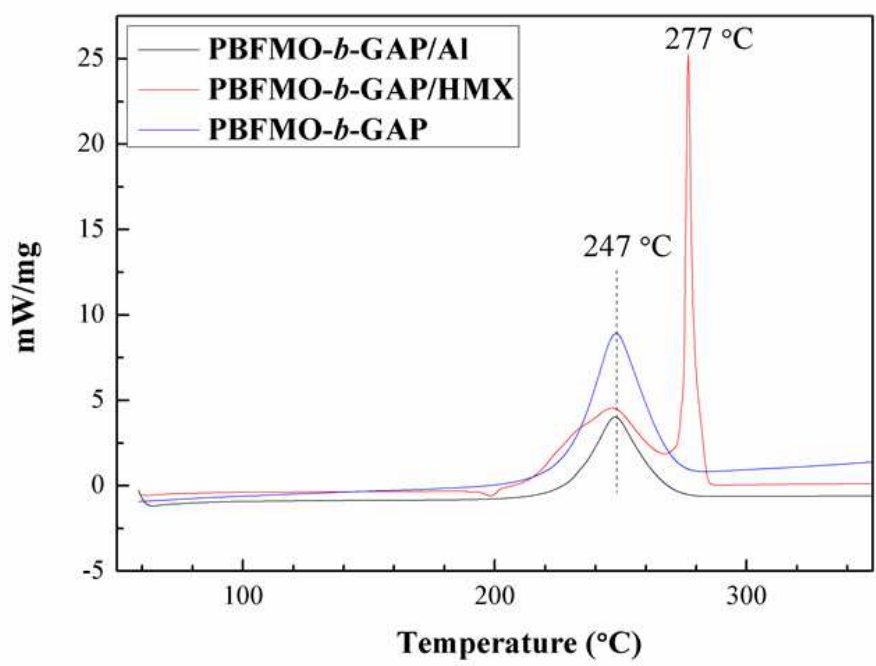

Figure 8. DSC curves of PBFMO- $b$-GAP, PBFMO- $b$-GAP/HMX complex and PBFMO- $b$-GAP/Al complex.

\subsection{Cook-off test}

The cook-off experiments were used to study the thermal performance of PBFMO- $b$-GAP and $\mathrm{Al}^{36-38}$. As shown in Figure 9, the cook-off curves showed initial linear calefactive and impetuously calefactive ultimately lead to linear calefactive again. The impetuously calefactive of inner 
temperature can be contribute to the samples start to exothermic decomposition, the release gas reactive with $\mathrm{Al}$ and give more exothermal. Generally, the integral of outer and inner temperature curves can evaluate the heat release due to the reaction between elastomers and Al. In this work, the integral of PBFMO- $b$-GAP/Al compositions showed a remarkable increase than the control group. It implied that the PBFMO- $b$-GAP can efficiently react with $\mathrm{Al}$ and release significantly more heat ${ }^{39}$.
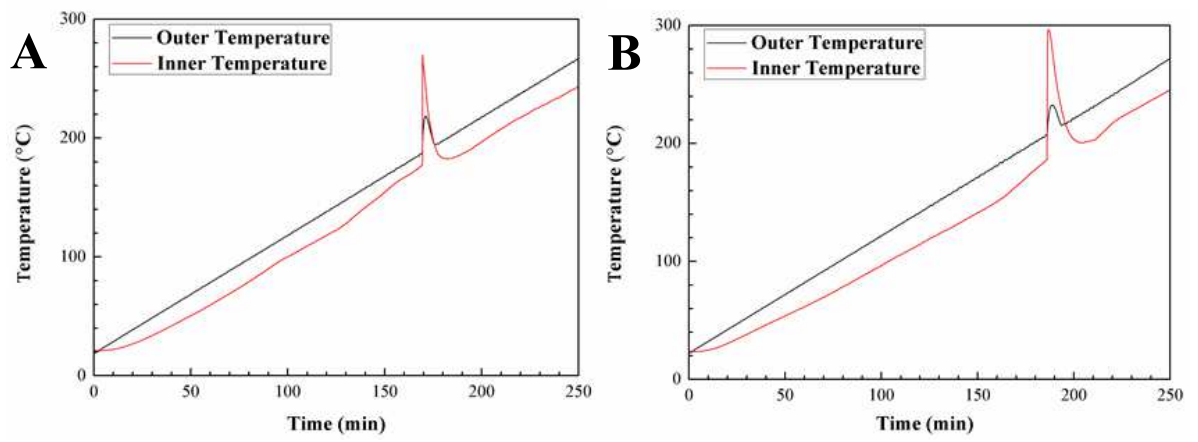

Figure 9. Cook-off curves of GAP-ETPE/Al (A) and PBFMO- $b-\mathrm{GAP} / \mathrm{Al}$ (B)

\section{Conclusions}

In conclusions, a copolyurethane binder, PBFMO- $b$-GAP, was synthesized on GAP as a soft segment and TDI extended PBFMO as hard segment. From FT-IR, NMR, and GPC results, the PBFMO-b-GAP was synthesized successfully via a prepolymer process. The drop weight test and XPS results indicated that the introduction of fluoropolymer can evidently reduce the sensitivity of PBFMO- $b$-GAP polyurethane. The PBFMO- $b$-GAP showed an enhanced tensile strength of 5.75 MPa with a breaking elongation of $1660 \%$, and the tensile strength of PBFMO- $b$-GAP films increased with an increase of the PBFMO content. The DSC and TGA-DTG curves indicated that PBFMO- $b$-GAP have adequate resistance to thermal decomposition up to $200^{\circ} \mathrm{C}$ and begin to decompose gradually at about $230^{\circ} \mathrm{C}$, and also have a good compatibility with $\mathrm{Al}$ and $\mathrm{HMX}$. Cook-off results implied the PBFMO- $b$-GAP can effectively react with Al and release relatively 
more quantity of heat. All these results indicated that PBFMO- $b$-GAP might serve as a potential energetic binder in propellant formulations.

\section{Methods}

209 Materials GAP with molecular weight of $3500 \mathrm{~g} \mathrm{~mol}^{-1}$ and hydroxy value of $0.9 \%$ was provided 210 from the Liming Chemical Engineering Research and Design Institute of Luoyang. 211 2,2,2-trifluoroethanol, 2,2-bisbromomethyl-3-bromo-propan-1-ol, butane diol (BDO), BF 3 -etherate 212 and dibutyltindilaurate (DBTDL) were purchased from J\&K scientific Ltd. (Shanghai). Toluene 213 diisocyanate (TDI), N,N-dimethylformamide (DMF), dichloromethane (DCM) and ethanol were 214 supplied by Chengdu Kelong Chemical Reagents Company. 1,2-dichloroethane was obtained from 215 Chengdu Jinshan Chemical Reagent Company. $\mathrm{BDO}$ and $\mathrm{BF}_{3}$-dimethyl ether were distilled under 216 reduced pressure prior to use. All solvents for the reactions were analytical grade and were dried 217 before use.

218 Synthesis of [3,3-bis(2,2,2-trifluoroethoxymethyl)oxetane] glycol (BFMO) As shown in 219 Scheme 1, BFMO was synthesized according to the literature procedure in two steps ${ }^{40}$. First step was 220 the synthesis of 3,3-bisbromomethyloxetane (BBMO). A mixture of 221 2,2-bisbromomethyl-3-bromo-propan-1-ol, $\mathrm{NaOH}, \mathrm{TBAB}$ and $\mathrm{DCM}$ was stirred at $40{ }^{\circ} \mathrm{C}$ for $8 \mathrm{~h}$. 222 And then, the organic layer was separated, died with $\mathrm{MgSO}_{4}$, and concentrated at atmospheric 223 pressure to remove DCM. The residue was distilled under reduce pressure and the fraction of $94^{\circ} \mathrm{C} / 4$ $224 \mathrm{mmHg}$ was collected to give the purified BBMO as a colorless liquid (yield, 70\%). Second step was 225 the conversion of BBMO to BFMO, a typical compound synthesis are described below. BBMO, 226 2,2,2-trifluoroethanol, $\mathrm{KOH}$ and phase-transfer catalyst TBAB were charged into $100 \mathrm{~mL}$ three-necked round-bottom flask. The reaction mixture was stirred at $85{ }^{\circ} \mathrm{C}$ for $24 \mathrm{~h}$. After the 
reaction, the organic phase was separated, washed with distilled water and died over anhydrous $\mathrm{MgSO}_{4}$. The BFMO was obtained by distillation and the fraction of $86{ }^{\circ} \mathrm{C} / 4 \mathrm{~mm} \mathrm{Hg}$ was collected (yield, 81.6\%).

Polymerization of PBFMO The PBFMO was synthesized through cationic ring-opening polymerization of BFMO. A typical reaction procedure was as follows: $\mathrm{BDO}, \mathrm{BF}_{3}$-etherate and dried methylene chloride were charged into a three necked flask fitted with a thermometer under argon and left stirred for $1 \mathrm{~h}$. BFMO was added into the mixture drop by drop within a period of $8 \mathrm{~h}$, and the reaction mixture was then left under stirred for an additional $24 \mathrm{~h}$. After then, the reaction was stopped by the addition of $1 \%$ sodium bicarbonate solution. The organic phase was washed with distilled water and concentrated by vacuum evaporation to acquire a white, wax polymer PBFMO (yield, 92.4\%).GPC analysis: $M_{\mathrm{n}}=6840 \mathrm{~g} \mathrm{~mol}^{-1}, \mathrm{PDI}=1.22$ (polystyrene standards).

Synthesis of PBFMO-b-GAP copolyurethane PBFMO- $b$-GAP copolyurethane was synthesized via a prepolymer process using PBFMO and GAP as raw materials, TDI as coupling agent. A typical reaction procedure was as follows: Exactly GAP, PBFMO, and freshly distilled 1,2-dichloroethane were placed in a $250 \mathrm{~mL}$ four-neck flask equipped with a condenser, mechanical stirrer and thermometer under high purity argon $(99.99 \%)$ and then heated to $60^{\circ} \mathrm{C}$. TDI and DBTDL was then dissolved in $20 \mathrm{~mL}$ 1,2-dichloroethane and added dropwise into the reaction solution. After stirring for an additional $2 \mathrm{~h}$, the reaction mixture was poured into $400 \mathrm{~mL}$ ethanol. The polymer was precipitated and separated out, dried in vacuum at $50^{\circ} \mathrm{C}$ for $24 \mathrm{~h}$ to obtain PBFMO- $b$-GAP copolyurethane (yield, 97\%).

Measurements FTIR spectra were measured on a Bruker Tensor 27 instrument (KBr pellet) in the range of 4000-400 $\mathrm{cm}^{-1}$. Nuclear magnetic resonance (NMR) spectra were recorded on a Bruker 500 
$\mathrm{MHz}$ instrument with $\mathrm{CDCl}_{3}$ as the solvent and tetramethylsilane as the internal standard. GPC was conducted on a Waters GPC, using tetrahydrofuran and polystyrene standards as the mobile phase and for calibration, respectively. A method of drop hammer impact sensitivity test for big-bill explosive was developed. The weight of the hammer was $5 \mathrm{~kg}$ and the drop height was between $0 \sim$ $1.29 \mathrm{~m}$. The size of big-bill explosive was $\varphi 20 \mathrm{~mm} \times 5 \mathrm{~mm}$ and its weight was $2.8 \mathrm{~g}$. XPS analysis was performed using a Sigmaprobe instrument (ThermoElectron Corp., UK) equipped with a nonmonochromatic Al KR $(\mathrm{hv}=1486.6 \mathrm{eV})$ source at a power of $300 \mathrm{~W}$. Mechanical properties of all the elastomers films were measured on an AG-X Plus testing machine (Shimadzu, Japan) with a tensile rate of $50 \mathrm{~mm} \mathrm{~min}^{-1}$. The testing films were cut into strips with a width of $10 \mathrm{~mm}$ and a distance between testing marks was $30 \mathrm{~mm}$, and kept at $0 \%$ humidity for 7 days before measurement. A mean value of five replicates from each film was taken. SEM observation was carried out on a temperature range from the room temperature to $250{ }^{\circ} \mathrm{C}$. In the slow cook-off test, we put 
272 cord, meanwhile the equal heating components of PBFMO- $b$-GAP was heated with an intelligent 273 temperature controller to adjust the heating rate. Samples were well sealed, and the thermocouples 274 were utilized to obtain their temperature vs time curves. Those experimental results were 275 synthetically analyzed to the reactivity of the PBFMO- $b$-GAP/Al complex under slow heating 276 stimulation.

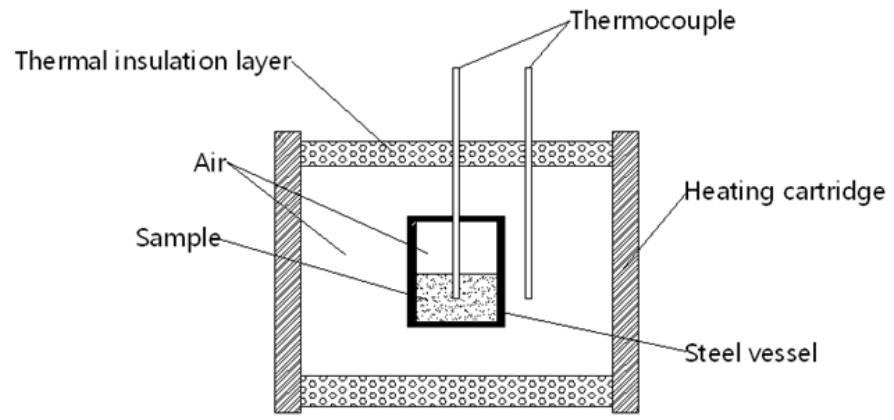

Scheme 2. Schematic geometry of cook-off test

\section{References}

1. Cheng, T. Review of novel energetic polymers and binders - high energy propellant ingredients for the new space race. Des. Monomers Polym. 22 1, 54-65 (2019).

2. Bodaghi, A. \& Shahidzadeh, M. Synthesis and characterization of new PGN based reactive oligomeric plasticizers for glycidyl azide polymer. Propellants Explos. Pyrotech. 43 4, 364-370 (2018).

3. Wang, Q., Wang, L., Zhang, X. \& Mi, Z. Thermal stability and kinetic of decomposition of nitrated HTPB. J. Hazard. Mater. 172 2-3, 1659-1664 (2009).

4. Hafner, S., Keicher, T. \& Klapoetke, T. M. Copolymers based on GAP and 1,2-Epoxyhexane as Promising Prepolymers for Energetic Binder Systems. Propellants Explos. Pyrotech. 43 2, 126-135 (2018). 
through anionic co-)polymerization of glycidyl azide. Nat. Commun. 10, 293-301 (2019).

6. Frankel, M. B., Grant, L. R. \& Flanagan, J. E. Historical development of glycidyl azide polymer. Journal of J.

Propul. Power 8 3, 560-563 (1992).

7. Murali Mohan, Y., Mani, Y. \& Mohana Raju, K. Synthesis of azido polymers as potential energetic propellent binders. Des. Monomers Polym. 9 3, 201-36 (2006).

8. Selim, K., Ozkar, S. \& Yilmaz, L. Thermal characterization of glycidyl azide polymer GAP.and GAP-based binders

15. Zhang, Z. et al. Synthesis and characterization of novel energetic thermoplastic elastomers based on glycidyl azide 
313 16. Lee, I., Reed, R. R., Brady, V. L. \& Finnegan, S. A. Energy release in the reaction of metal powders with fluorine containing polymers. J. Therm. Anal. 49 3, 1699-1705 (1997).

17. Lee, J. H., Kim, S. J., Park, J. S. \& Kim, J. H. Energetic Al/Fe2O3/PVDF composites for high energy release: Importance of polymer binder and interface. J. Therm. Anal. 24 10, 909-914 (2016).

18. Dattelbaum, D. M. et al. Equation of state and high pressure properties of a fluorinated terpolymer: THV 500. J. Appl. Phys. 104 11, 113525-113535 (2008).

19. McCollum, J., Pantoya, M. L. \& Iacono, S. T. Activating aluminum reactivity with fluoropolymer coatings for 11032-11039 (2016). 
control of an in situ Cu-free azide-alkyne 1,3-dipolar cycloaddition reaction. Polym. Chem. 9 45, 5452-5461 (2018).

27. Jin, B. et al. Synthesis, characterization, thermal stability and sensitivity properties of new energetic polymers-PVTNP-g-GAPs crosslinked polymers. Polymers 8 1, 10-23 (2016).

28. Shmatova, O. I. \& Nenajdenko, V. G. Tetrazole-substituted five, six, and seven-membered cyclic amines bearing perfluoroalkyl groups - efficient synthesis by azido-ugi reaction. Eur. J. Org. Chem. 2013 28, 6397-6403 (2013).

29. Sarangapani, R., Reddy, S. T. \& Sikder, A. K. Molecular dynamics simulations to calculate glass transition temperature and elastic constants of novel polyethers. J. Mol. Graphics Modell. 57, 114-121 (2015).

30. Cai, T., Yang, W. J., Neoh, K. G. \& Kang, E. T. Polyvinylidene fluoride.membranes with hyperbranched antifouling and antibacterial polymer brushes. Ind. Eng. Chem. Res. 51 49, 15962-15973 (2012).

31. Malkappa, K. \& Jana, T. Simultaneous improvement of tensile strength and elongation: An unprecedented observation in the case of hydroxyl terminated polybutadiene polyurethanes. Ind. Eng. Chem. Res. 52 36, $12887-12896$ (2013).

32. Landsem, E. et al. Isocyanate-free and dual curing of smokeless composite rocket propellants. Propellants Explos. Pyrotech. 38 1, 75-86 (2013).

33. You, J. S., Kweon, J. O., Kang, S. C. \& Noh, S. T. A kinetic study of thermal decomposition of glycidyl azide polymer GAP)-based energetic thermoplastic polyurethanes. Macromol. Res. 18 12, 1226-1232 (2010).

34. Pei, J. F. et al. Compatibility study of BAMO-GAP copolymer with some energetic materials. J. Therm. Anal. Calorim. 124 3, 1301-1307 (2016).

35. Li, Y., Li, J., Ma, S. \& Luo, Y. Compatibility, mechanical and thermal properties of GAP/PEO-co-THF.blends obtained upon a urethane-curing reaction. Polym. Bull. 74 11, 4607-4618 (2017).

36. Ding, X. Y., Shu, Y. J., Xu, H. T. \& Chen, Z. Q. Study on thermal behaviour of AP/LiBH4 energetic system. Propellants Explos. Pyrotech. 43 3, 267-273 (2018). 
38. Chen, L., Ma, X., Lu, F. \& Wu, J. Y. Investigation of the cook-off processes of hmx-based mixed explosives. Cent. Eur. J. Energ. Mat. 11 2, 199-218 (2014).

39. Xu, M. et al. Fluorinated glycidyl azide polymers as potential energetic binders. $R S C A d v .775,47271-47278$ (2017).

40. Jiang, W. C., Huang, Y. G., Gu, G. T., Meng, W. D. \& Qing, F. L. A novel waterborne polyurethane containing short 2304-2309 (2006).

\section{Acknowledgements}

367 The authors gratefully acknowledge the financial support from the China Postdoctoral 368 Science Foundation (2016M592851).

\section{Author contributions}

370 Conceptualization, M.X. and X.L.; Methodology, M.X., X.L. and H.M.; Investigation, M.X.; 371 Resources, X.L. Q.Z. and H.M.; Writing-original draft preparation, M.X.; Writing-review and 372 editing, M.X., Q.Z. and H.M.; Supervision, M.X., N.L. and Z.G.; Funding acquisition, M.X.

\section{Competing interests}

374 The authors declare no competing interests. 
Figures

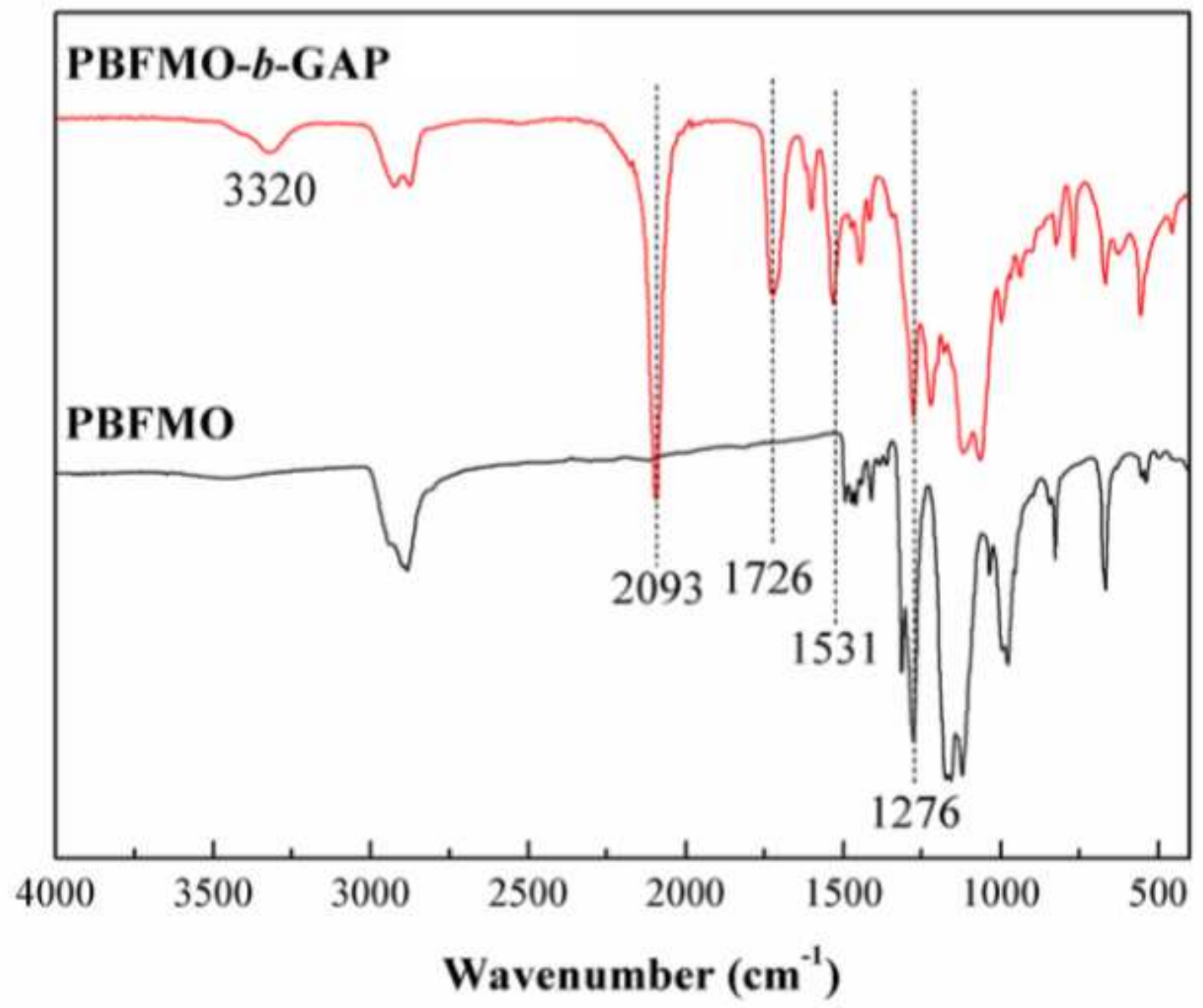

Figure 1

FTIR spectra of (A) PBFMO and (B) PBFMO-b-GAP copolyurethane.
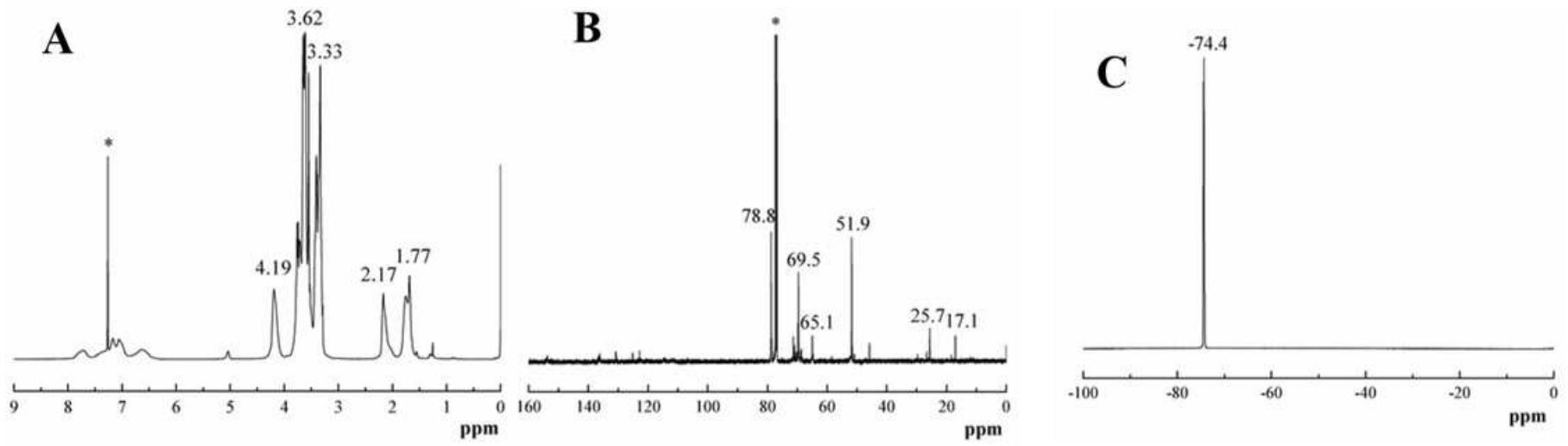

Figure 2 
H-NMR spectra (A),13C-NMR spectra (B) and19F-NMR spectra (C) of PBFMO-b-GAP in CDCl3.
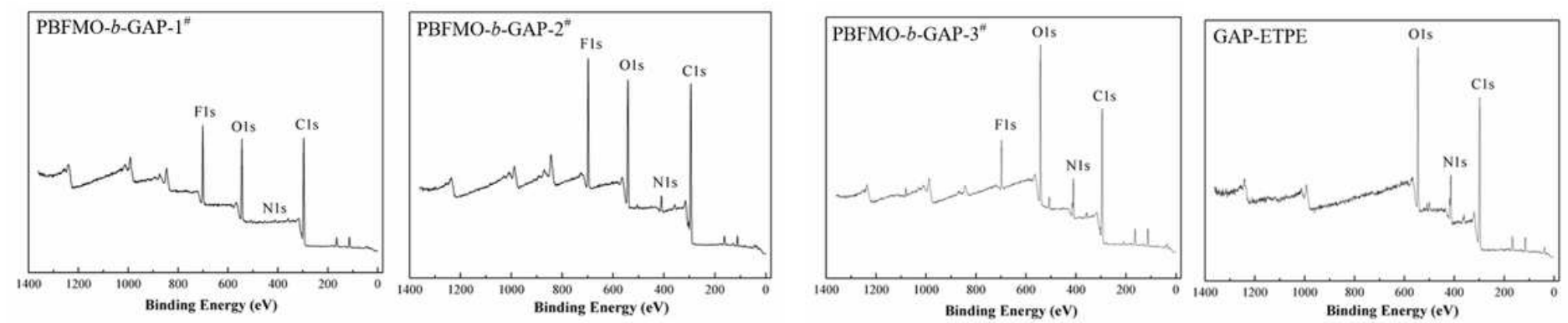

\section{Figure 3}

XPS curves of the gels prepared from GAP based polyurethanes

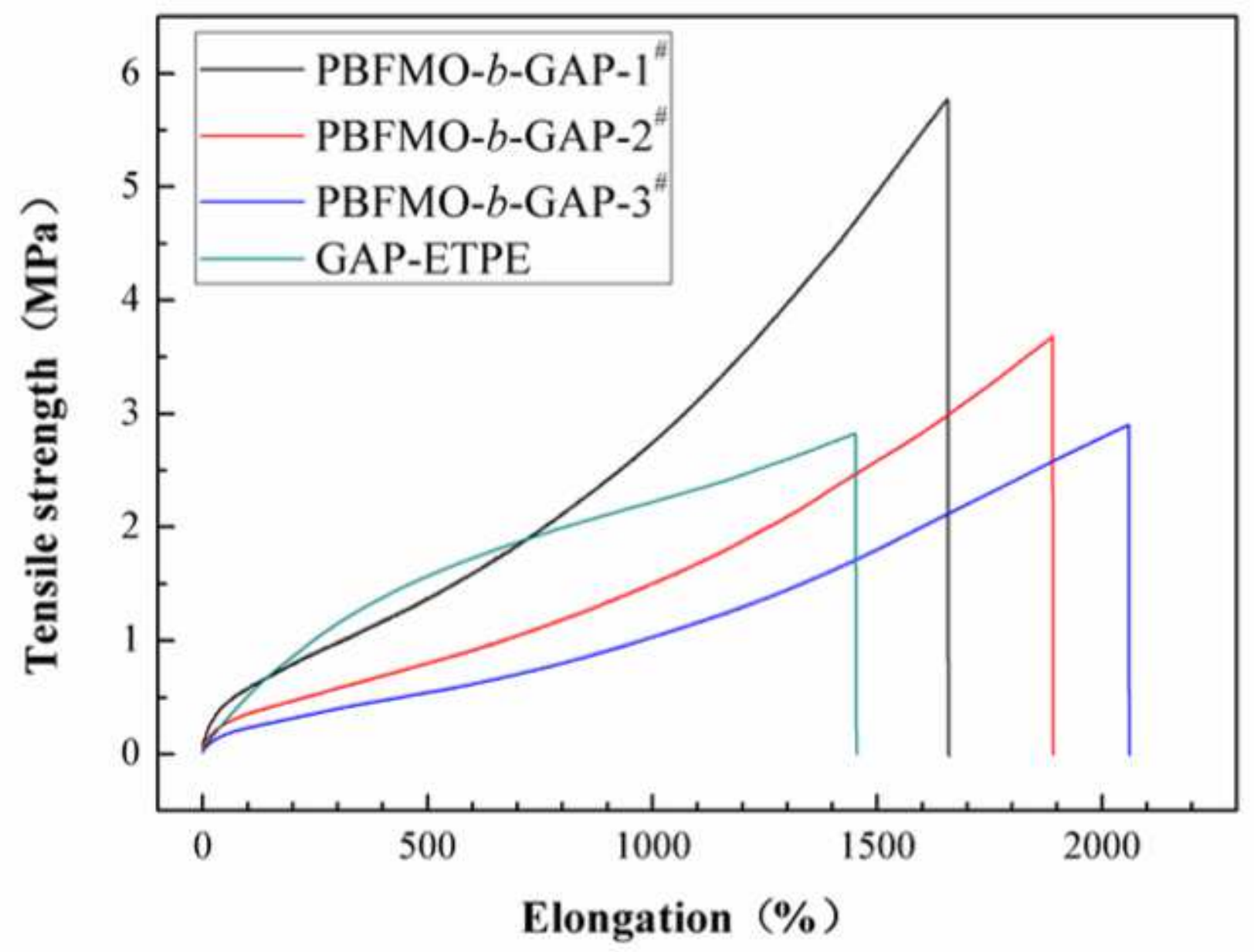

Figure 4

Tensile testing of polymer samples prepared from PBFMO-b-GAP copolyurethane. 

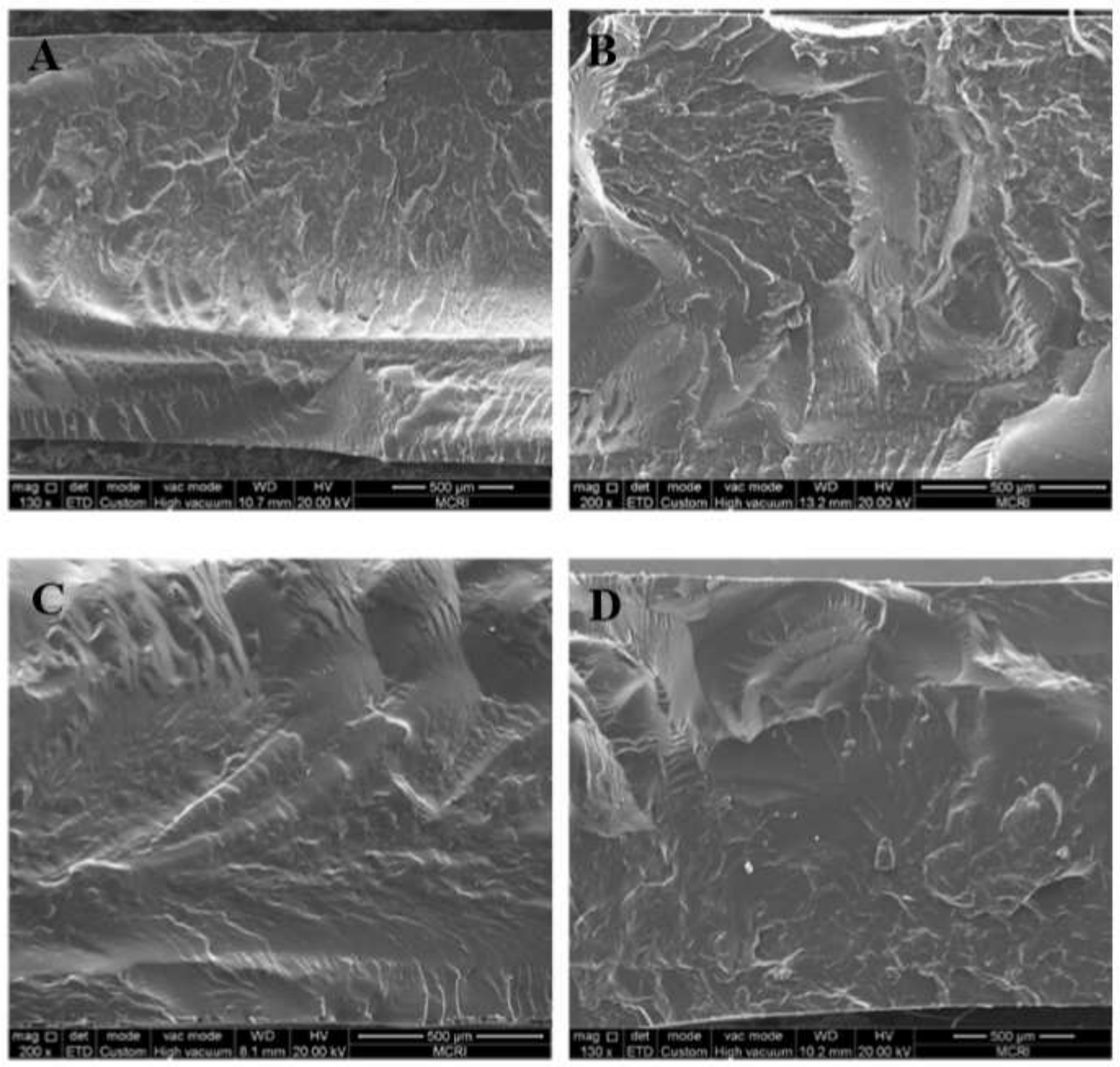

\section{Figure 5}

SEM images for the fracture surface of the gels prepared from (A) PBFMO-b-GAP-1\#, (B) 150 PBFMO-bGAP-2\#, (C) PBFMO-b-GAP-3\# and (D) GAP-ETPE. 


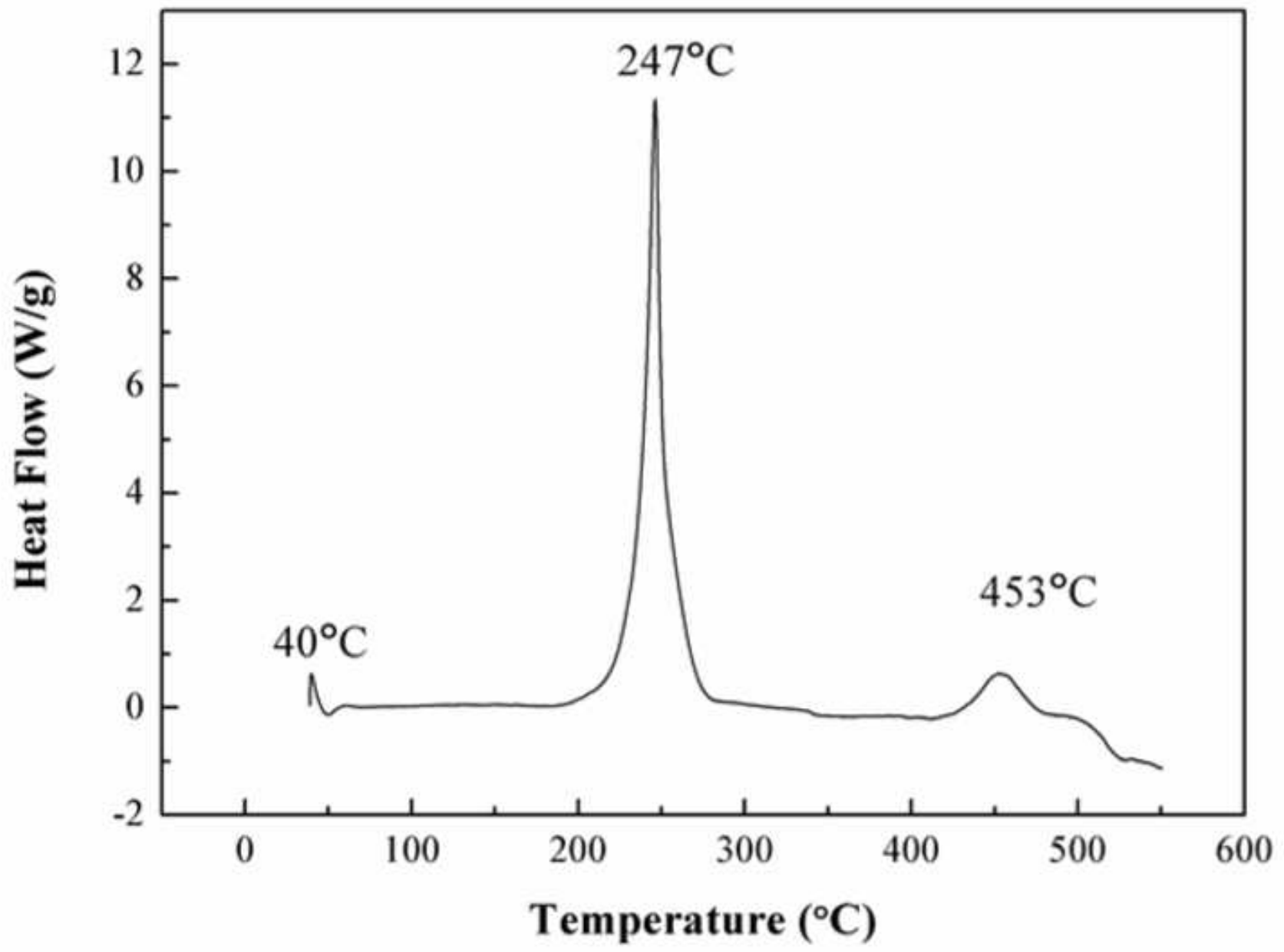

Figure 6

DSC curve of PBFMO-b-GAP copolyurethane. 


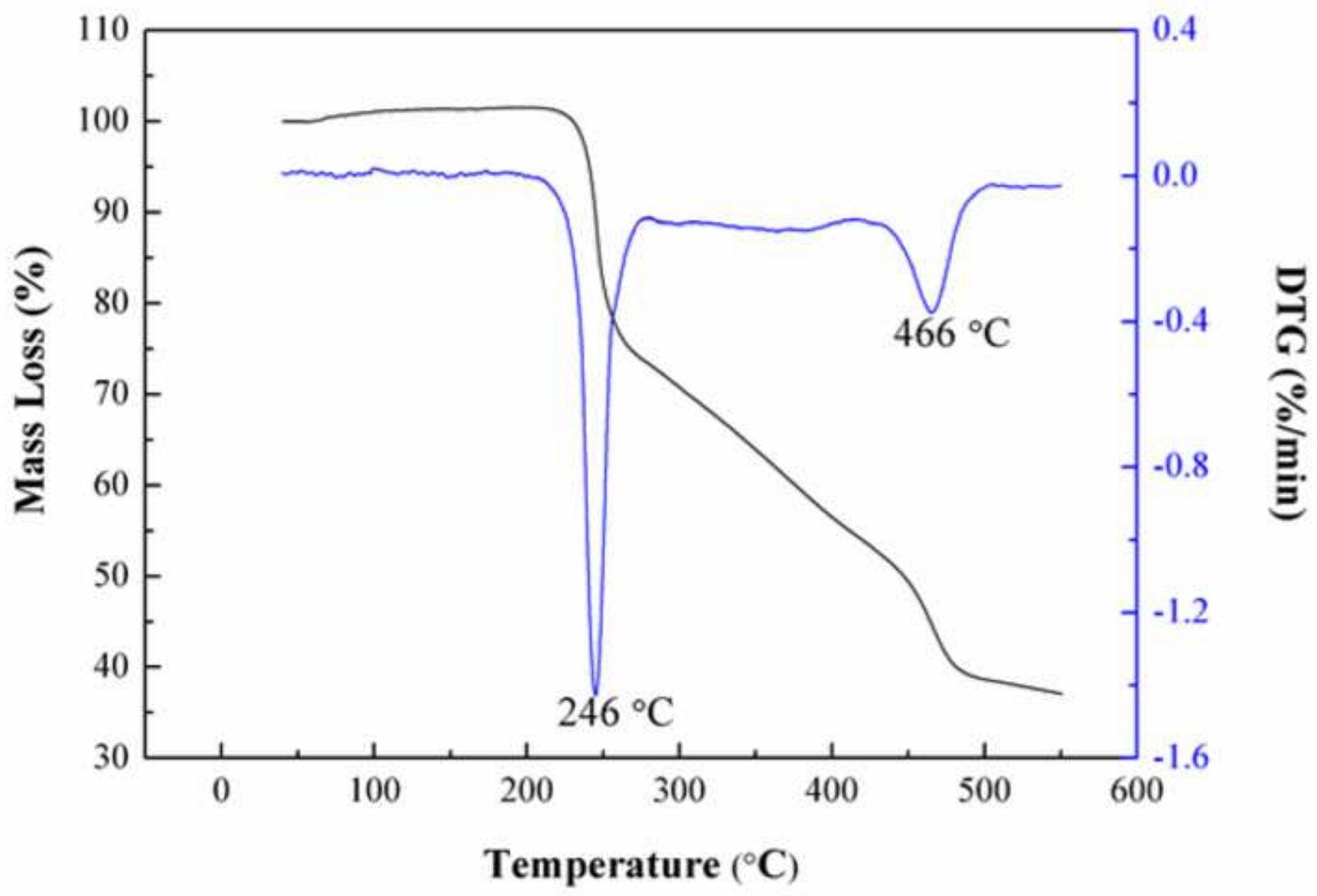

Figure 7

TG/DTG curves of PBFMO-b-GAP copolyurethane. 


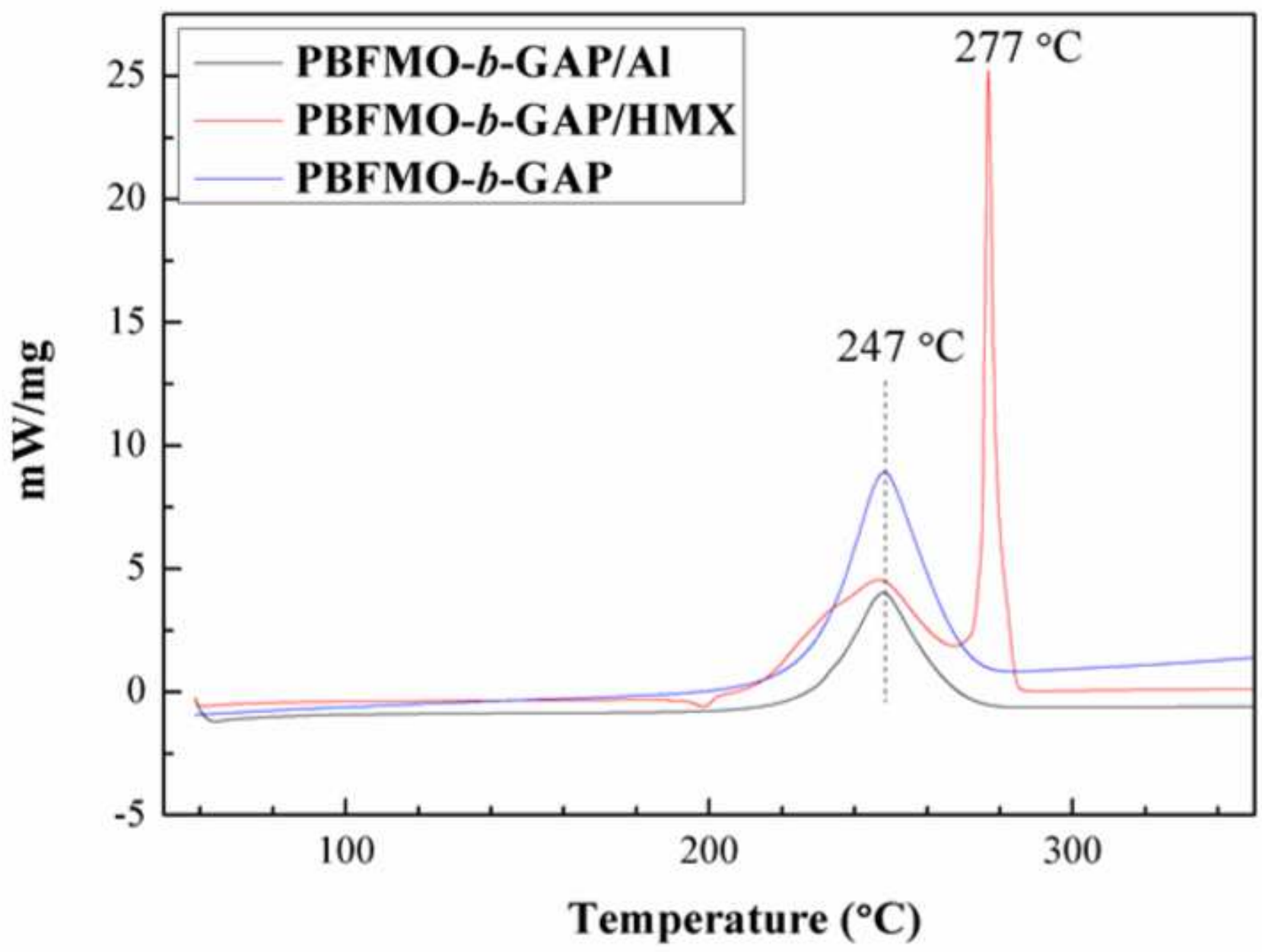

Figure 8

DSC curves of PBFMO-b-GAP, PBFMO-b-GAP/HMX complex and PBFMO-b-GAP/Al complex.
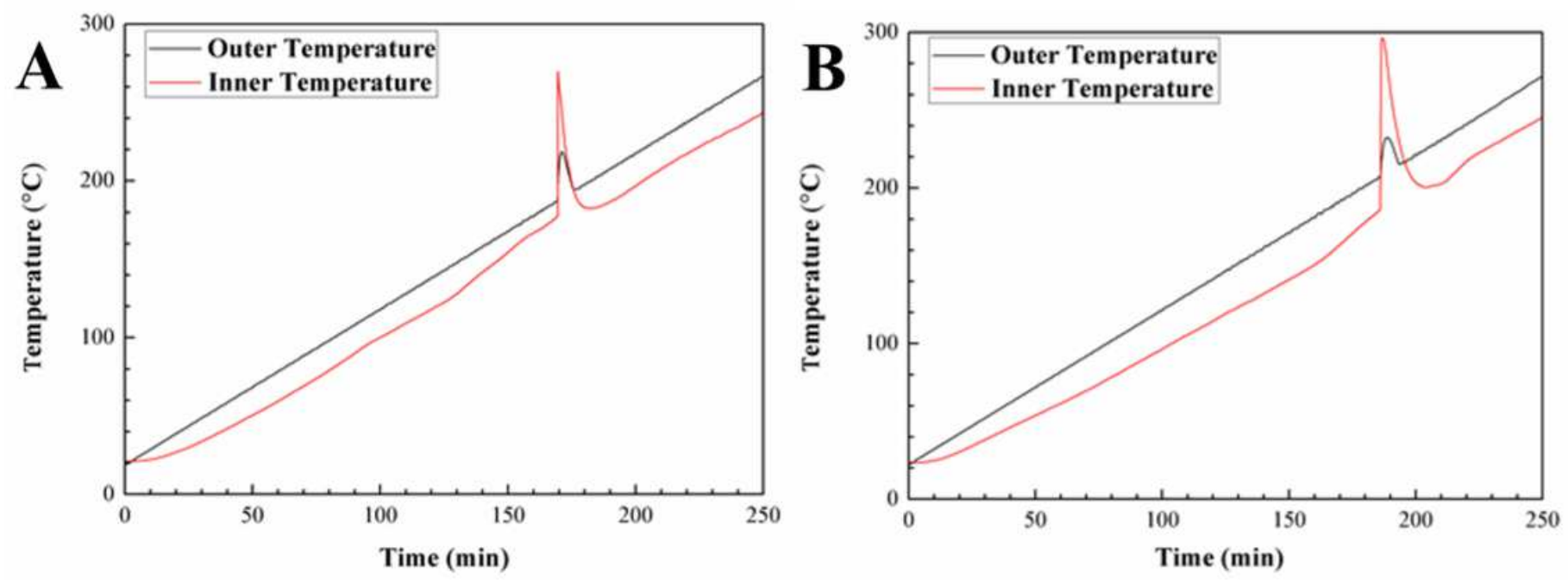

Figure 9 
Cook-off curves of GAP-ETPE/Al (A) and PBFMO-b-GAP/Al (B)

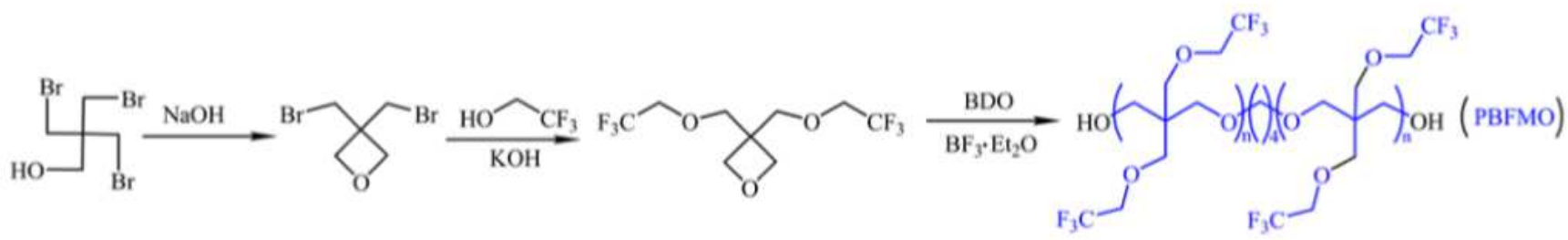

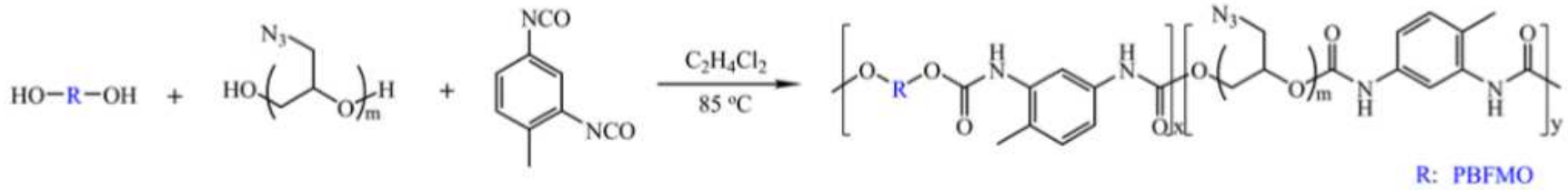

\section{Figure 10}

The synthesis route of PBFMO-b-GAP copolyurethane

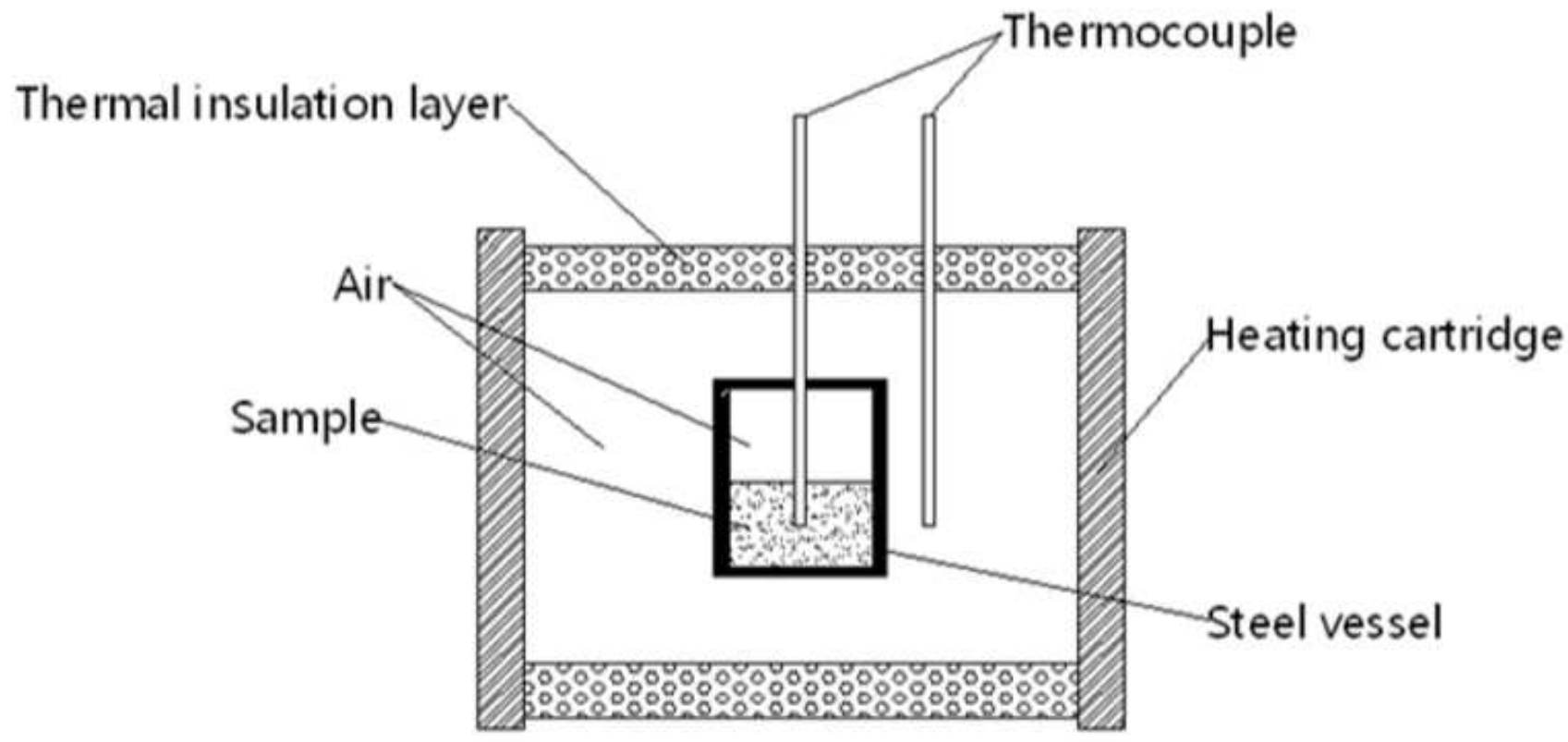

\section{Figure 11}

Schematic geometry of cook-off test 